\title{
Asymptotically flat spacetimes in three-dimensional higher spin gravity
}

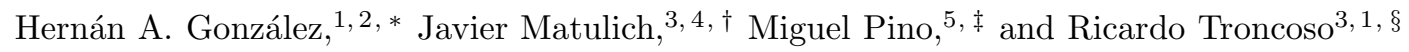 \\ ${ }^{1}$ Universidad Andrés Bello, Av. República 440, Santiago, Chile. \\ ${ }^{2}$ Facultad de Física, Pontificia Universidad Católica de Chile, Casilla 306, Santiago 22, Chile. \\ ${ }^{3}$ Centro de Estudios Científicos (CECs), Casilla 1469, Valdivia, Chile. \\ ${ }^{4}$ Departamento de Física, Universidad de Concepción, Casilla, 160-C, Concepción, Chile. \\ ${ }^{5}$ Departamento de Física, Universidad de Santiago de Chile, \\ Av. Ecuador 3493, Estación Central, Santiago, Chile.
}

\begin{abstract}
A consistent set of asymptotic conditions for higher spin gravity in three dimensions is proposed in the case of vanishing cosmological constant. The asymptotic symmetries are found to be spanned by a higher spin extension of the $\mathrm{BMS}_{3}$ algebra with an appropriate central extension. It is also shown that our results can be recovered from the ones recently found for asymptotically $\mathrm{AdS}_{3}$ spacetimes by virtue of a suitable gauge choice that allows to perform the vanishing cosmological constant limit.
\end{abstract}

\section{INTRODUCTION}

Higher spin gravity [1 1 ] has recently motivated a flurry of activity. In the case of $d \geq 4$ dimensions, the consistency of the theory necessarily requires the presence of a negative cosmological constant, which naturally makes it to be appealing in the context of holographic dualities [4, 5] (For recent reviews see e.g., 6 [11]). The three-dimensional case becomes particularly interesting in order to acquire a deeper understanding of the subject. Indeed, in this case the generic theory turns out to be exceptionally simpler, since it can be described as a standard field theory in terms of a Chern-Simons action. Moreover, it can be further simplified because it admits a consistent truncation that characterizes the dynamics of a finite number of higher spin gauge fields [12, 13]. Remarkably, the theory in $d=3$ can also be formulated in the case of vanishing cosmological constant $\Lambda$. Therefore, this latter specific case naturally becomes worth of special attention, since one may naturally expect that it could unveil some clues about the elusive construction of higher spin gravity around flat spacetime in higher dimensions.

The purpose of this work is to propose a precise set of asymptotic conditions for higher spin gravity in three dimensions in the case of $\Lambda=0$. In the next section, the theory is revisited in some detail, for simplicity, in the case of spins $s=2,3$. Section 【II is devoted to present the asymptotically flat conditions, including the analysis of their asymptotic symmetry group. The corresponding conserved charges are also shown to span a higher spin extension of the $\mathrm{BMS}_{3}$ algebra with a nontrivial central extension. In section IV we show that these results can be recovered from the corresponding ones in the case of asymptotically $\mathrm{AdS}_{3}$ spacetimes [14, 15]. This task is performed through a particular "decoupling" gauge choice, that allows the straightforward application of the vanishing $\Lambda$ limit. The procedure allows extending the asymptotically flat conditions to the case of spins $s \geq 2$. Finally, the discussion is carried out in section $\nabla$

Note added: While this work was being typed, ref. [16] was posted on the arXiv, whose results overlap with some of ours in sections III, IV]

\footnotetext{
${ }^{*}$ Electronic address: hdgonzal-at-uc.cl

$\dagger$ Electronic address: matulich-at-cecs.cl

¥Electronic address: miguel.pino.r-at-usach.cl

$\S$ Electronic address: troncoso-at-cecs.cl
} 


\section{HIGHER SPIN GRAVITY IN 3D WITH VANISHING COSMOLOGICAL CONSTANT}

Higher spin gravity in three dimensions can be formulated in terms of a Chern-Simons action [12, 13], given by,

$$
I[A]=\frac{k}{4 \pi} \int\left\langle A d A+\frac{2}{3} A^{3}\right\rangle .
$$

For the sake of simplicity, in this section we discuss in some detail the case of spins $s=2,3$. In this case, the gauge field $A=A_{\mu} d x^{\mu}$ can be written as (see e.g., [14, 15])

$$
A=\omega^{a} J_{a}+e^{a} P_{a}+W^{a b} J_{a b}+E^{a b} P_{a b}
$$

where the set $\left\{J_{a}, P_{a}, J_{a b}, P_{a b}\right\}$ spans the gauge group, and the generators $P_{a b}, J_{a b}$ are assumed to be symmetric and traceless. As explained in [15], in the case of vanishing cosmological constant, the generators fulfill a generalization of the Poincaré algebra, given by ${ }^{1}$

$$
\begin{aligned}
& {\left[J_{a}, J_{b}\right]=\epsilon_{a b c} J^{c}, \quad\left[P_{a}, J_{b}\right]=\epsilon_{a b c} P^{c}, \quad\left[P_{a}, P_{b}\right]=0,} \\
& {\left[J_{a}, J_{b c}\right]=\epsilon_{a(b}^{m} J_{c) m}, \quad\left[J_{a}, P_{b c}\right]=\epsilon_{a(b}^{m} P_{c) m},} \\
& {\left[P_{a}, J_{b c}\right]=\epsilon_{a(b}^{m} P_{c) m}, \quad\left[P_{a}, P_{b c}\right]=0,} \\
& {\left[J_{a b}, J_{c d}\right]=-\left(\eta_{a(c} \epsilon_{d) b m}+\eta_{b(c} \epsilon_{d) a m}\right) J^{m}, \quad\left[J_{a b}, P_{c d}\right]=-\left(\eta_{a(c} \epsilon_{d) b m}+\eta_{b(c} \epsilon_{d) a m}\right) P^{m},} \\
& {\left[P_{a b}, J_{c d}\right]=-\left(\eta_{a(c} \epsilon_{d) b m}+\eta_{b(c} \epsilon_{d) a m}\right) P^{m}, \quad\left[P_{a b}, P_{c d}\right]=0,}
\end{aligned}
$$

the level is related to the Newton constant according to $k=\frac{1}{4 G}$, and the bracket $\langle\cdots\rangle$ in (11) stands for a non-degenerate invariant bilinear product, whose only nonvanishing components are given by ${ }^{2}$

$$
\left\langle P_{a} J_{b}\right\rangle=\eta_{a b}, \quad\left\langle P_{a b} J_{c d}\right\rangle=\eta_{a c} \eta_{b d}+\eta_{a d} \eta_{c b}-\frac{2}{3} \eta_{a b} \eta_{c d}
$$

Therefore, the action (11) reduces to

$$
I[e, \omega, E, W]=\frac{k}{2 \pi} \int\left[e^{a}\left(d \omega_{a}+\frac{1}{2} \epsilon_{a b c} \omega^{b} \omega^{c}+2 \epsilon_{a b c} W^{b d} W_{d}^{c}\right)+2 E^{a b}\left(d W_{a b}+2 \epsilon_{c d a} \omega^{c} W_{b}^{d}\right)\right]
$$

up to a boundary term, and the field equations read

$$
\begin{aligned}
d e^{a}+\epsilon^{a b c} \omega_{b} e_{c}+4 \epsilon^{a b c} E_{b d} W_{c}^{d} & =0, \\
d \omega^{a}+\frac{1}{2} \epsilon^{a b c} \omega_{b} \omega_{c}+2 \epsilon^{a b c} W_{b d} W_{c}^{d} & =0, \\
d E^{a b}+\epsilon^{c d(a \mid} \omega_{c} E_{d}{ }^{\mid b)}+\epsilon^{c d(a \mid} e_{c} W_{d}{ }^{\mid b)} & =0 \\
d W^{a b}+\epsilon^{c d(a \mid} \omega_{c} W_{d}{ }^{\mid b)} & =0 .
\end{aligned}
$$

The fields $e^{a}, E^{a b}$, and $\omega^{a}, W^{a b}$ are interpreted as a generalization of the dreibein and the spin connection, respectively; so that the metric and the spin-3 field can be constructed from the only quadratic and cubic

\footnotetext{
1 This algebra can be obtained from a contraction of two copies of $\operatorname{sl}(3)$ (see appendix $\mathrm{A}$ ).

2 This is related to the fact that the algebra (3) admits a Casimir operator given by $C=P^{a} J_{a}+\frac{1}{2} P^{a b} J_{a b}$.
} 
combinations of the generalized dreibein that are invariant under Lorentz-like transformations generated by $J_{a}, J_{a b}$, i.e.

$$
\begin{aligned}
d s^{2} & =\left(\eta_{a b} e_{\mu}^{a} e_{\nu}^{b}+2 \eta_{a c} \eta_{b d} E_{\mu}^{a b} E_{\nu}^{c d}\right) d x^{\mu} d x^{\nu}, \\
\varphi & =\left(\eta_{a c} \eta_{b d} e_{\mu}^{a} e_{\nu}^{b} E_{\rho}^{c d}-\frac{4}{3} \eta_{c d} \eta_{a g} \eta_{b h} E_{\mu}^{g c} E_{\nu}^{h d} E_{\rho}^{a b}\right) d x^{\mu} d x^{\nu} d x^{\rho} .
\end{aligned}
$$

Note that if $e_{\mu}^{a}$ is assumed to be invertible, then in the case of $E^{a b}=0$, the field equations imply that $W^{a b}$ also vanishes, and hence, General Relativity in vacuum is recovered.

\section{ASYMPTOTICALLY FLAT SPACETIMES ENDOWED WITH HIGHER SPIN FIELDS}

It has been shown that asymptotically flat spacetimes in General Relativity with vanishing cosmological constant in three dimensions enjoy similar features as the ones exhibited by asymptotically $\mathrm{AdS}_{3}$ geometries [17]. Indeed, the asymptotic symmetry group is infinite dimensional [18], and its algebra, so-called $\mathrm{BMS}_{3}$, also acquires a nontrivial central extension [19, 20]. It has also been recently shown that these results can be recovered from a suitable Penrose-like limit of the asymptotic behaviour of the metric on $\mathrm{AdS}_{3}$ [21]. The $\mathrm{BMS}_{3}$ algebra turns out to be isomorphic to the Galilean conformal algebra in two dimensions $\left(\mathrm{GCA}_{2}\right)$ [22], which becomes relevant in the context of non-relativistic holography.

It is then natural to look for suitable asymptotic conditions in the case of higher spin gravity, being such that they fulfill the following requirements:

(i) Reducing to the ones of [19, 20] when the higher spin fields are switched off, and

(ii) In presence of higher spin fields, they should correspond to the vanishing cosmological constant limit of the asymptotically $\mathrm{AdS}_{3}$ conditions of [14, 15, 23, 24].

In this section the analysis is explicitly carried out in the simplest case of spins $s=2,3$; since the generic case of higher spins is shown to be one of the outputs of section IV]

For the theory under consideration, gauge fields satisfying the previous requirements, are proposed to possess the following asymptotic form ${ }^{3}$ :

$$
\begin{aligned}
A=\left(\frac{1}{2} \mathcal{M} d u-d r+\left(\mathcal{J}+\frac{u}{2} \partial_{\phi} \mathcal{M}\right) d \phi\right) P_{0}+d u P_{1}+ & r d \phi P_{2}+\frac{1}{2} \mathcal{M} d \phi J_{0}+d \phi J_{1} \\
& +\left(\mathcal{W} d u+\left(\mathcal{V}+u \partial_{\phi} \mathcal{W}\right) d \phi\right) P_{00}+\mathcal{W} d \phi J_{00}
\end{aligned}
$$

where $r, \phi$ correspond to the radial and angular coordinates, respectively, $u$ is a null coordinate that plays the role of time, and $\mathcal{M}, \mathcal{J}, \mathcal{W}$ and $\mathcal{V}$ stand for arbitrary functions of $\phi$. According to (99), it is apparent that condition (i) is fulfilled in the case of $\mathcal{W}=\mathcal{V}=0$; while condition (ii) is explicitly shown to be satisfied in the next section.

The asymptotic symmetries correspond to gauge transformations generated by a Lie-algebra-valued parameter

$$
\lambda=\rho^{a} P_{a}+\eta^{a} J_{a}+\xi^{a b} P_{a b}+\Lambda^{a b} J_{a b},
$$

that preserves the form of (9), i.e.,

$$
\delta A=d \lambda+[A, \lambda]=\mathcal{O}(A)
$$

\footnotetext{
${ }^{3}$ Hereafter, our conventions are such that we assume a non-diagonal Minkowski metric in tangent space, whose only nonvanishing components are given by $\eta_{01}=\eta_{10}=\eta_{22}=1$, and the Levi-Civita symbol fulfills $\epsilon_{012}=1$.
} 
One then finds that $\lambda=\lambda(\epsilon, y, w, v)$ depends on four independent functions of the angular coordinate, being defined as

$$
\rho^{1}=\epsilon+u y^{\prime}, \quad \eta^{1}=y, \quad \xi^{11}=w+u v^{\prime}, \quad \Lambda^{11}=v
$$

where prime denotes the derivative with respect to $\phi$. The remaining components of $\lambda$ are given in appendix B.

The arbitrary functions appearing in the asymptotic form of the gauge field are found to transform according to the following rules

$$
\begin{aligned}
\delta \mathcal{M} & =y \mathcal{M}^{\prime}+2 y^{\prime} \mathcal{M}-2 y^{\prime \prime \prime}+4\left(2 v \mathcal{W}^{\prime}+3 v^{\prime} \mathcal{W}\right) \\
\delta \mathcal{J} & =y \mathcal{J}^{\prime}+2 y^{\prime} \mathcal{J}+\frac{1}{2} \epsilon \mathcal{M}^{\prime}+\epsilon^{\prime} \mathcal{M}-\epsilon^{\prime \prime \prime}+2\left(2 w \mathcal{W}^{\prime}+3 w^{\prime} \mathcal{W}+2 v \mathcal{V}^{\prime}+3 v^{\prime} \mathcal{V}\right) \\
\delta \mathcal{W} & =y \mathcal{W}^{\prime}+3 y^{\prime} \mathcal{W}-\frac{1}{6} v \mathcal{M}^{\prime \prime \prime}-\frac{3}{4} v^{\prime} \mathcal{M}^{\prime \prime}-\frac{5}{4} v^{\prime \prime} \mathcal{M}-\frac{5}{6} v^{\prime \prime \prime} \mathcal{M}+\frac{2}{3}\left(v \mathcal{M} \mathcal{M}^{\prime}+v^{\prime} \mathcal{M}^{2}\right)+\frac{1}{6} v^{(5)}, \\
\delta \mathcal{V} & =y \mathcal{V}^{\prime}+3 y^{\prime} \mathcal{V}+\epsilon \mathcal{W}^{\prime}+3 \epsilon^{\prime} \mathcal{W}-\frac{5}{3} v^{\prime \prime \prime} \mathcal{J}-\frac{5}{2} v^{\prime \prime} \mathcal{J}^{\prime}-\frac{3}{2} v^{\prime} \mathcal{J}^{\prime \prime}-\frac{1}{3} v \mathcal{J}^{\prime \prime \prime}+\frac{4}{3} v(\mathcal{J} \mathcal{M})^{\prime} \\
& +\frac{8}{3} v^{\prime} \mathcal{M} \mathcal{J}+\frac{2}{3}\left(w \mathcal{M} \mathcal{M}^{\prime}+w^{\prime} \mathcal{M}^{2}\right)-\frac{1}{6} w \mathcal{M}^{\prime \prime \prime}-\frac{3}{4} w^{\prime} \mathcal{M}^{\prime \prime}-\frac{5}{4} w^{\prime \prime} \mathcal{M}^{\prime}-\frac{5}{6} w^{\prime \prime \prime} \mathcal{M}+\frac{1}{6} w^{(5)},
\end{aligned}
$$

which allows to find the precise form of the asymptotic symmetry algebra. This can be readily carried out as follows. The variation of the global charges that correspond to the asymptotic symmetries spanned by $\lambda$, in the canonical approach [25], is given by

$$
\delta Q[\lambda]=\frac{k}{2 \pi} \int\left\langle\lambda \delta A_{\phi}\right\rangle d \phi
$$

Once the variation of the charges is evaluated on the asymptotic form of the connection (9), it is found that it becomes linear in the variation of the fields, so that it can be integrated as

$$
Q[\epsilon, y, w, v]=\frac{k}{4 \pi} \int[\epsilon \mathcal{M}+2 y \mathcal{J}+4(w \mathcal{W}+v \mathcal{V})] d \phi
$$

Their algebra can then be straightforwardly obtained from the variation of the fields since, as explained in [26],$\delta_{\lambda_{2}} Q\left[\lambda_{1}\right]=\left\{Q\left[\lambda_{1}\right], Q\left[\lambda_{2}\right]\right\}$. Indeed, as expected, the asymptotic symmetries associated to $\epsilon(\phi)$ and $y(\phi)$ span the $\mathrm{BMS}_{3}$ algebra with the same central charge as in the case of General Relativity in three dimensions [19]. In this case, the Poisson brackets read

$$
\left\{Q\left(\epsilon_{1}, y_{1}\right), Q\left(\epsilon_{2}, y_{2}\right)\right\}=Q\left(\epsilon_{[1,2]}, y_{[1,2]}\right)+K\left[\epsilon_{1}, \epsilon_{2}, y_{1}, y_{2}\right]
$$

where the parameters $\epsilon_{[1,2]}$ and $y_{[1,2]}$ are given by

$$
\begin{aligned}
\epsilon_{[1,2]} & =\epsilon_{1} y_{2}^{\prime}-\epsilon_{2} y_{1}^{\prime}-\epsilon_{1}^{\prime} y_{2}+\epsilon_{2}^{\prime} y_{1}, \\
y_{[1,2]} & =y_{1} y_{2}^{\prime}-y_{1}^{\prime} y_{2}
\end{aligned}
$$

and the central charge $K$ is

$$
K\left[\epsilon_{1}, \epsilon_{2}, y_{1}, y_{2}\right]=\frac{k}{2 \pi} \int\left[\epsilon_{1}^{\prime} y_{2}^{\prime \prime}-\epsilon_{2}^{\prime} y_{1}^{\prime \prime}\right] d \phi
$$

Expanding in Fourier modes $P_{n}=Q\left(\epsilon=e^{i n \phi}\right)$ and $J_{n}=Q\left(y=e^{i n \phi}\right)$, the algebra acquires the form

$$
\begin{aligned}
i\left\{P_{n}, P_{m}\right\} & =0, \\
i\left\{J_{n}, J_{m}\right\} & =(n-m) J_{n+m}, \\
i\left\{J_{n}, P_{m}\right\} & =(n-m) P_{n+m}+k n^{3} \delta_{m+n} .
\end{aligned}
$$


The brackets of the $\mathrm{BMS}_{3}$ generators with the remaining charges associated to $w(\phi)$ and $v(\phi)$, are given by

$$
\left\{Q\left(\epsilon_{1}, y_{1}\right), Q\left(w_{2}, v_{2}\right)\right\}=Q\left(w_{[1,2]}, v_{[1,2]}\right)
$$

where $w_{[1,2]}$ and $v_{[1,2]}$ read

$$
\begin{aligned}
& w_{[1,2]}=\epsilon_{1} v_{2}^{\prime}-2 \epsilon_{1}^{\prime} v_{2}+w_{2}^{\prime} y_{1}-2 w_{2} y_{1}^{\prime}, \\
& v_{[1,2]}=y_{1} v_{2}^{\prime}-2 v_{2} y_{1}^{\prime} .
\end{aligned}
$$

Therefore, the corresponding Fourier modes, $W_{n}=Q\left(w=e^{i n \phi}\right)$ and $V_{n}=Q\left(v=e^{i n \phi}\right)$, fulfill

$$
\begin{aligned}
i\left\{P_{n}, W_{m}\right\} & =0, \\
i\left\{J_{n}, W_{m}\right\} & =(2 n-m) W_{n+m}, \\
i\left\{P_{n}, V_{m}\right\} & =(2 n-m) W_{n+m}, \\
i\left\{J_{n}, V_{m}\right\} & =(2 n-m) V_{n+m},
\end{aligned}
$$

from which, one verifies that the charges $W_{n}$ and $V_{n}$ correspond to spin-3 generators. Finally, the brackets of the spin-3 generators turn out to be given by

$$
\begin{aligned}
i\left\{W_{n}, W_{m}\right\} & =0 \\
i\left\{W_{n}, V_{m}\right\} & =\frac{1}{3}\left[\frac{8}{k}(n-m) \sum_{j=-\infty}^{\infty} P_{j} P_{n+m-j}+(n-m)\left(2 n^{2}+2 m^{2}-m n\right) P_{m+n}+k n^{5} \delta_{m+n}\right], \\
i\left\{V_{n}, V_{m}\right\} & =\frac{1}{3}\left[\frac{16}{k}(n-m) \sum_{j=-\infty}^{\infty} P_{j} J_{n+m-j}+(n-m)\left(2 n^{2}+2 m^{2}-m n\right) J_{m+n}\right],
\end{aligned}
$$

so that the nonlinearity of the algebra becomes manifest. Note that the structure of the brackets of the spin-3 generators is reminiscent of the one of $\mathrm{BMS}_{3}$, in the sense that $W_{n}$ can be regarded as a higher spin extension of the supertranslations $P_{n}$, and the central term appears in the crossed bracket.

In sum, the commutation relations (19), (23) and (24) provide the searched for higher spin extension of the $\mathrm{BMS}_{3}$ algebra.

It is interesting to express the asymptotic form of the connection $A$ in (9), in terms of the metric and the spin-3 field, which read

$$
\begin{aligned}
d s^{2} & =\mathcal{M} d u^{2}-2 d u d r+2\left(\mathcal{J}+\frac{u}{2} \partial_{\phi} \mathcal{M}\right) d u d \phi+r^{2} d \phi^{2} \\
\varphi & =\left(\mathcal{W} d u+\left(\mathcal{V}+u \partial_{\phi} \mathcal{W}\right) d \phi\right) d u^{2}
\end{aligned}
$$

respectively. From this, it can be explicitly seen that for our gauge choice, the fields decouple, in the sense that the metric does not acquire any kind of backreaction from the spin-3 field, neither the spin-3 field does from the metric. Furthermore, unlike the case of $U(1)$ fields coupled to General Relativity (see e.g., [27]), the spin-3 field does not contribute to neither the energy nor the angular momentum. Hence, in spite of these unusual features between the coupling of the spin-3 field with the metric, it is amusing to verify that the charges still fulfill a rather nontrivial algebra that mixes the asymptotic form of both fields in a nonlinear form. It is also worth pointing out that a similar effect is known to occur for a class of configurations in the case of scalar fields non-minimally coupled to gravity [28]. The next section is devoted to show that our asymptotic conditions fulfill requirement (ii), as well as to provide the extension of these results to the case of spins $s \geq 2$.

\section{VANISHING COSMOLOGICAL CONSTANT LIMIT OF $\mathrm{ADS}_{3}$ BOUNDARY CONDITIONS}

Higher spin gravity on $\mathrm{AdS}_{3}$ is described through the difference of two Chern-Simons actions, whose gauge fields correspond to two copies of a single algebra [12, 13]. The asymptotic behavior of the fields, 
in this case, has been discussed in [14, 15, 23, 24], for $s l(N)$ and $h s(\lambda)$. In the "highest weight gauge", the asymptotic form of the gauge fields can be written as

$$
A^{ \pm}=b_{ \pm}^{-1} a^{ \pm} b_{ \pm}+b_{ \pm}^{-1} d b_{ \pm}
$$

where $b_{ \pm}=e^{ \pm \log (r / l) L_{0}}$, and

$$
a^{ \pm}= \pm\left(L_{ \pm 1}-\Xi_{ \pm} L_{\mp 1}-W_{ \pm} W_{\mp 2}+\cdots\right) d x^{ \pm} .
$$

Here $\Lambda=-\frac{1}{l^{2}}, L_{i}$ describes the generators of $s l(2)$ in the principal embedding, $\Xi_{ \pm}$and $W_{ \pm}$correspond to arbitrary functions of $x^{ \pm}=\frac{t}{l} \pm \phi$, and the dots stand for additional terms involving the higher spin generators $(s>3)$ of highest weight.

For our purposes, it is convenient to make a different gauge choice, so that the asymptotic form of the gauge fields read

$$
A^{ \pm}=g_{ \pm}{ }^{-1} a^{ \pm} g_{ \pm}+g_{ \pm}{ }^{-1} d g_{ \pm}
$$

with

$$
\begin{aligned}
& g_{+}=b_{+} e^{-\log \left(\sqrt{2} \frac{r}{l}\right) L_{0}} e^{\frac{r}{\sqrt{2} l} L_{-1}}, \\
& g_{-}=b_{-} e^{-\log \left(\frac{1}{2 \sqrt{2}} \frac{r}{l}\right) L_{0}} e^{\frac{r}{\sqrt{2} l} L_{-1}} e^{\sqrt{2} \frac{l}{r} L_{1}} .
\end{aligned}
$$

Therefore, the components of the connections are given by

$$
A^{ \pm}=\frac{r}{l} d x^{ \pm} L_{0}^{ \pm} \pm \frac{1}{\sqrt{2}}\left[\frac{d r}{l}+\left(\frac{r^{2}}{2 l^{2}}-2 \Xi_{ \pm}\right) d x^{ \pm}\right] L_{-1}^{ \pm} \pm \frac{d x^{ \pm}}{\sqrt{2}} L_{1}^{ \pm} \mp 2 d x^{ \pm}\left(W_{ \pm} W_{-2}^{ \pm}+\cdots\right) .
$$

As it is explained below, it is convenient to make the change $t=u$, since in this gauge, $u$ becomes a null coordinate. In order to explore some of the features of this gauge choice, it is instructive to discuss the case of $\operatorname{sl}(3)$ with more detail. In this case, it has been shown that the asymptotic symmetries are generated by two copies of the $W_{3}$ algebra [14], 15], defined by

$$
\begin{aligned}
i\left\{\mathcal{L}_{n}^{ \pm}, \mathcal{L}_{m}^{ \pm}\right\} & =(n-m) \mathcal{L}_{n+m}^{ \pm}+\frac{k l}{2} n^{3} \delta_{m+n}, \\
i\left\{\mathcal{L}_{n}^{ \pm}, \mathcal{W}_{m}^{ \pm}\right\} & =(2 n-m) \mathcal{W}_{n+m}^{ \pm} \\
i\left\{\mathcal{W}_{n}^{ \pm}, \mathcal{W}_{m}^{ \pm}\right\} & =\frac{1}{3}\left[\frac{16}{k l}(n-m) \sum_{j=-\infty}^{\infty} \mathcal{L}_{j}^{ \pm} \mathcal{L}_{n+m-j}^{ \pm}+(n-m)\left(2 n^{2}+2 m^{2}-m n\right) \mathcal{L}_{m+n}^{ \pm}+\frac{k l}{2} n^{5} \delta_{m+n}\right] .
\end{aligned}
$$

It is also useful to change the basis according to

$$
\begin{gathered}
L_{-1}^{ \pm}=-\sqrt{2} J_{0}^{ \pm}, \quad L_{0}^{ \pm}=J_{2}^{ \pm}, \quad L_{1}^{ \pm}=\sqrt{2} J_{1}^{ \pm}, \\
W_{-2}^{ \pm}=-2 T_{00}^{ \pm}, \quad W_{-1}^{ \pm}=\sqrt{2} T_{02}^{ \pm}, \quad W_{0}^{ \pm}=-T_{22}^{ \pm}, \quad W_{1}^{ \pm}=-\sqrt{2} T_{12}^{ \pm}, \quad W_{2}^{ \pm}=-2 T_{11}^{ \pm},
\end{gathered}
$$

where the generators $T_{a b}$ are traceless, followed by

$$
J_{a}^{ \pm}=\frac{J_{a} \pm l P_{a}}{2} \quad T_{a b}^{ \pm}=\frac{J_{a b} \pm l P_{a b}}{2},
$$

so that the full gauge field reads

$$
\begin{aligned}
A=A^{+}+A^{-}= & \left(\frac{1}{2} \mathcal{M} d \phi+\frac{\mathcal{N}}{l^{2}} d u-\frac{r^{2}}{2 l^{2}} d \phi\right) J_{0}+d \phi J_{1}+\frac{r}{l^{2}} d u J_{2} \\
& +\left(-d r+\frac{1}{2} \mathcal{M} d u+\mathcal{N} d \phi-\frac{r^{2}}{2 l^{2}} d u\right) P_{0}+d u P_{1}+r d \phi P_{2} \\
& +\left(\mathcal{W} d \phi+\frac{2}{l^{2}} \mathcal{Q} d u\right) J_{00}+(\mathcal{W} d u+2 \mathcal{Q} d \phi) P_{00}
\end{aligned}
$$


Here, the arbitrary functions of $u$ and $\phi$ have been conveniently redefined as

$$
\begin{aligned}
& \mathcal{M}=2\left(\Xi_{+}+\Xi_{-}\right), \quad \mathcal{N}=l\left(\Xi_{+}-\Xi_{-}\right), \\
& \mathcal{W}=2\left(W_{+}+W_{-}\right), \quad \mathcal{Q}=l\left(W_{+}-W_{-}\right),
\end{aligned}
$$

and then, the chirality conditions now read

$$
\begin{array}{ll}
\partial_{u} \mathcal{M}=\frac{2}{l^{2}} \partial_{\phi} \mathcal{N} & 2 \partial_{u} \mathcal{N}=\partial_{\phi} \mathcal{M} \\
\partial_{u} \mathcal{W}=\frac{2}{l^{2}} \partial_{\phi} \mathcal{Q} & 2 \partial_{u} \mathcal{Q}=\partial_{\phi} \mathcal{W}
\end{array}
$$

From eq. (33) the generalized dreibein can be directly read, so that the asymptotic form of the metric and the spin-3 field are recovered from eqs. (7), (8), which leads to

$$
\begin{aligned}
d s^{2} & =\left(-\frac{r^{2}}{l^{2}}+\mathcal{M}\right) d u^{2}-2 d u d r+2 \mathcal{N} d u d \phi+r^{2} d \phi^{2}, \\
\varphi & =(\mathcal{W} d u+2 \mathcal{Q} d \phi) d u^{2} .
\end{aligned}
$$

One then verifies that, unlike what occurs in the highest weight gauge, and as in the previous section, for our gauge choice the fields decouple, in the sense that the spin-3 field does not generate any back-reaction on the metric. Moreover, since the canonical charges, written in appendix [C] do not change under the gauge transformation generated by $g_{ \pm}$in eqs. (26), (27), the spin-3 field gives no contribution to the energy and angular momentum.

One of the main advantages of expressing the asymptotic form of the connection in our gauge choice is that the vanishing cosmological constant limit can be taken in a straightforward way. This can be seen as follows. In the limit $l \rightarrow \infty$, the chirality conditions (36), (37) imply that

$$
\mathcal{M}=\mathcal{M}(\phi), \quad \mathcal{N}=\mathcal{J}(\phi)+\frac{u}{2} \partial_{\phi} \mathcal{M}, \quad \mathcal{W}=\mathcal{W}(\phi), \quad \mathcal{Q}=\frac{1}{2}\left(\mathcal{V}(\phi)+u \partial_{\phi} \mathcal{W}\right)
$$

Hence, replacing (38) into the connection (33), it is simple to verify that for $l \rightarrow \infty$ the asymptotically flat conditions in eq. (9), are recovered. This is also the case for the global charges which, as explained in appendix [C] after a suitable mapping of the functions that parametrize the asymptotic symmetries, reduce to (14) in the limit.

It is also very simple to check that the asymptotic symmetries, described by two copies of the $W_{3}$ algebra (29), reduce to the spin-3 extension of $\mathrm{BMS}_{3}$. Indeed, by redefining the generators as

$$
\begin{aligned}
P_{n} & =\frac{1}{l}\left(\mathcal{L}_{n}^{+}+\mathcal{L}_{-n}^{-}\right), \\
J_{n} & =\mathcal{L}_{n}^{+}-\mathcal{L}_{-n}^{-}, \\
W_{n} & =\frac{1}{l}\left(\mathcal{W}_{n}^{+}+\mathcal{W}_{-n}^{-}\right), \\
V_{n} & =\mathcal{W}_{n}^{+}-\mathcal{W}_{-n}^{-},
\end{aligned}
$$

the asymptotic symmetry algebra in the flat case, given by eqs. (19), (23), (24) is recovered once we take the limit $l \rightarrow \infty$.

An additional benefit of this procedure, is that the asymptotic behavior of higher spin gravity with $\Lambda=0$, for spins $s \geq 2$, can be readily obtained from the connection in the asymptotically $\mathrm{AdS}_{3}$ case with our gauge choice, as in eq. (28). Indeed, following the steps described above, asymptotically flat spacetimes in higher spin gravity with vanishing cosmological constant are found to be described by the following connection 


$$
\begin{aligned}
A=\left(\frac{1}{2} \mathcal{M} d u-d r\right. & \left.+\left(\mathcal{J}+\frac{u}{2} \partial_{\phi} \mathcal{M}\right) d \phi\right) P_{0}+d u P_{1}+r d \phi P_{2}+\frac{1}{2} \mathcal{M} d \phi J_{0}+d \phi J_{1} \\
& +\left(\mathcal{W}_{3} d u+\left(\mathcal{V}_{3}+u \partial_{\phi} \mathcal{W}_{3}\right) d \phi\right) P_{00}+\mathcal{W}_{3} d \phi J_{00} \\
& +\left(\mathcal{W}_{4} d u+\left(\mathcal{V}_{4}+u \partial_{\phi} \mathcal{W}_{4}\right) d \phi\right) P_{000}+\mathcal{W}_{4} d \phi J_{000} \\
& +\ldots
\end{aligned}
$$

where the arbitrary functions $\mathcal{M}, \mathcal{J}, \mathcal{W}_{3}, \mathcal{V}_{3}, \mathcal{W}_{4}, \mathcal{V}_{4}, \ldots$, depend only on $\phi$, and the generators $P_{a_{1} \cdots a_{s-1}}$, $J_{a_{1} \cdots a_{s-1}}$, are fully symmetric and traceless. The asymptotic symmetry algebra that corresponds to the higher spin extension of $\mathrm{BMS}_{3}$ is then obtained from the one from the asymptotically $\mathrm{AdS}_{3}$ case, spanned by two copies of $\mathrm{W}_{N}$, or $\mathrm{W}_{\infty}[\lambda]$, by redefining the generators as

$$
\begin{aligned}
P_{n} & =\frac{1}{l}\left(\mathcal{L}_{n}^{+}+\mathcal{L}_{-n}^{-}\right), \\
J_{n} & =\mathcal{L}_{n}^{+}-\mathcal{L}_{-n}^{-}, \\
W_{n}^{(3)} & =\frac{1}{l}\left(\mathcal{W}_{n}^{(3)+}+\mathcal{W}_{-n}^{(3)-}\right), \\
V_{n}^{(3)} & =\mathcal{W}_{n}^{(3)+}-\mathcal{W}_{-n}^{(3)-}, \\
W_{n}^{(4)} & =\frac{1}{l}\left(\mathcal{W}_{n}^{(4)+}+\mathcal{W}_{-n}^{(4)-}\right), \\
V_{n}^{(4)} & =\mathcal{W}_{n}^{(4)+}-\mathcal{W}_{-n}^{(4)-}, \\
& \ldots
\end{aligned}
$$

and then taking the limit $l \rightarrow \infty$.

\section{DISCUSSION}

The asymptotically $\mathrm{AdS}_{3}$ conditions of [14, 15, 23, 24], that extend the ones of Brown and Henneaux to the case of higher spin fields, have been shown to possess an interesting vanishing cosmological constant limit. As a consequence, this means that solutions of the field equations with $\Lambda<0$ that fulfill these conditions, can be consistently mapped to asymptotically flat ones that satisfy ours. Then, in particular, it is worth pointing out that the smoothed out conical defects and surpluses discussed in [29 31] fall within this category, i.e., they admit a consistent $l \rightarrow \infty$ limit that fulfills the field equations of higher spin gravity around flat space with the boundary conditions discussed here. It is then natural to expect that their holonomies around spacelike cycles remain trivial after the limit, but nonetheless, an explicit check of this claim would be worth to be done.

Note that, as it has been recently shown in [32], the fact that General Relativity possesses a set of asymptotic conditions fulfilling the $\mathrm{BMS}_{3}$ algebra, implies that the theory can be described in terms of a flat analogue of Liouville theory at null infinity, that, as shown in [33], corresponds to a suitable limit of its $\mathrm{AdS}_{3}$ counterpart [34]. This strongly suggests that a similar construction could be performed starting from the asymptotically flat conditions in presence of higher spin fields discussed here, which would naturally be identified with a flat analogue of Toda theory.

As an ending remark, it would be worth exploring whether the asymptotic conditions proposed here could be generalized in a consistent way with the asymptotic symmetries. In this sense, we would like pointing out that, at least in the case of spins $s=2,3$, a good starting point might be the generic solution of the field equations that fulfills the conditions

$$
\begin{gathered}
\omega_{u}^{a}=0, \quad e_{u}^{a}=\omega_{\phi}^{a}, \\
W_{u}^{a b}=0, \quad E_{u}^{a b}=W_{\phi}^{a b},
\end{gathered}
$$


which ensure that the action attains an extremum. The solution is given by

$$
\begin{aligned}
W^{a b} & =\mathcal{W}^{a b} d \phi, \\
E^{00} & =\mathcal{W}^{00} d u+\left(\mathcal{V}^{00}+u \partial_{\phi} \mathcal{W}^{00}-2 r \mathcal{W}^{02}\right) d \phi, \\
E^{01} & =\mathcal{W}^{01} d u+\left(\mathcal{V}^{01}+u \partial_{\phi} \mathcal{W}^{01}-r \mathcal{W}^{12}\right) d \phi, \\
E^{02} & =\mathcal{W}^{02} d u+\left(\mathcal{V}^{02}+u \partial_{\phi} \mathcal{W}^{02}+3 r \mathcal{W}^{01}\right) d \phi, \\
E^{11} & =\mathcal{W}^{11} d u+\left(\mathcal{V}^{11}+u \partial_{\phi} \mathcal{W}^{11}\right) d \phi, \\
E^{12} & =\mathcal{W}^{12} d u+\left(\mathcal{V}^{12}+u \partial_{\phi} \mathcal{W}^{12}+r \mathcal{W}^{11}\right) d \phi,
\end{aligned}
$$

where $\mathcal{W}^{a b}, \mathcal{V}^{a b}$ are arbitrary functions of $\phi$, while $e^{a}$ and $\omega^{a}$ are given by the ones of General Relativity with $\Lambda=0$.

\section{Acknowledgments}

We thank G. Barnich, M. Bañados, E. Bergshoeff, R. Canto, A. Campoleoni, A. Castro, C. Erices, J. Gamboa, M. Gary, D. Grumiller, M. Henneaux, C. Martínez, A. Pérez, P. Sundell, D. Tempo, and C. Troessaert for useful discussions and comments. J.M. thanks Conicyt for financial support. M.P. is partially funded by Conicyt, project 7912010045. R.T. thanks the Galileo Galilei Institute for Theoretical Physics, the Physique théorique et mathématique group of the Université Libre de Bruxelles, and the International Solvay Institutes for the kind hospitality. This research has been partially supported by Fondecyt grants $\mathrm{N}^{\circ} 1130658,1121031$. Centro de Estudios Científicos (CECs) is funded by the Chilean Government through the Centers of Excellence Base Financing Program of Conicyt.

\section{Appendix A: Higher spin extension of the Poincaré algebra as a contraction of $\operatorname{sl}(3) \oplus \operatorname{sl}(3)$}

The algebra (3) can be easily seen to arise from a contraction of two copies of $s l(3)$, whose generators fulfill

$$
\begin{aligned}
& {\left[J_{a}^{ \pm}, J_{b}^{ \pm}\right]=\epsilon_{a b c} J^{ \pm c}} \\
& {\left[J_{a}^{ \pm}, T_{b c}^{ \pm}\right]=\epsilon_{a(b}^{m} T_{c) m}^{ \pm}} \\
& {\left[T_{a b}^{ \pm}, T_{c d}^{ \pm}\right]=-\left(\eta_{a(c} \epsilon_{d) b m}+\eta_{b(c} \epsilon_{d) a m}\right) J^{ \pm m}}
\end{aligned}
$$

This can be performed by changing the basis of the $\operatorname{sl}(3) \oplus \operatorname{sl}(3)$ generators according to

$$
\begin{aligned}
& P_{a}=\frac{1}{l}\left(J_{a}^{+}-J_{a}^{-}\right), \quad J_{a}=J_{a}^{+}+J_{a}^{-}, \\
& P_{a b}=\frac{1}{l}\left(T_{a b}^{+}-T_{a b}^{-}\right), \quad J_{a b}=T_{a b}^{+}+T_{a b}^{-},
\end{aligned}
$$

so that after taking the limit $l \rightarrow \infty$, the algebra (3) is recovered.

\section{Appendix B: Lie-algebra-valued parameter components of the asymptotically flat symmetries}

The components of the Lie-algebra-valued parameter $\lambda=\lambda(\epsilon, y, w, v)$ in eq. (10), associated to the asymptotically flat symmetries, depend on four arbitrary functions of $\phi$ and its derivatives. They are 
given by

$$
\begin{aligned}
\rho^{0}= & -y^{\prime \prime \prime} u+\left(r+\frac{u}{2} \mathcal{M}\right) y^{\prime}+4 v u \mathcal{W}^{\prime}+\frac{1}{2} y u \mathcal{M}^{\prime}+4 u \mathcal{W} v^{\prime}-\epsilon^{\prime \prime}+y \mathcal{J} \\
& +\frac{1}{2} \mathcal{M} \epsilon+4 v \mathcal{V}+4 \mathcal{W} w \\
\rho^{1}= & \epsilon+u y^{\prime} \\
\rho^{2}= & r y-y^{\prime \prime} u-\epsilon^{\prime}, \\
\eta^{0}= & -y^{\prime \prime}+\frac{1}{2} \mathcal{M} y+4 \mathcal{W} v, \\
\eta^{1}= & y \\
\eta^{2}= & -y^{\prime} \\
\xi^{00}= & \frac{1}{6} u v^{(5)}-\frac{1}{3}(r+2 u \mathcal{M}) v^{\prime \prime \prime}-\frac{1}{6} u \mathcal{M}^{\prime \prime \prime} v-\frac{3}{4} u \mathcal{M}^{\prime \prime} v^{\prime}-\frac{5}{4} u \mathcal{M}^{\prime} v^{\prime \prime}+v\left(\frac{r}{3}+\frac{u}{2} \mathcal{M}\right) \mathcal{M}^{\prime} \\
& +\frac{1}{12}\left(10 r \mathcal{M}+3 \mathcal{M}{ }^{2} u\right) v^{\prime}+u \mathcal{W} y^{\prime}+\mathcal{W} \epsilon+y \mathcal{V}-\frac{4}{3} \mathcal{J} v^{\prime \prime}+J \mathcal{M} v-\frac{1}{3} v \mathcal{J}^{\prime \prime}-\frac{1}{6} \mathcal{M}^{\prime \prime} w \\
& -\frac{7}{12} \mathcal{M}^{\prime} w^{\prime}-\frac{2}{3} \mathcal{M} w^{\prime \prime}-\frac{7}{6} v^{\prime} \mathcal{J}^{\prime}+\frac{1}{4} \mathcal{M}^{2} w+\frac{1}{6} w^{(4)}+u \mathcal{W}^{\prime} y, \\
\xi^{01}= & \frac{1}{2} r v^{\prime}-\frac{1}{6} v^{\prime \prime \prime} u-\frac{1}{6} w^{\prime \prime}+\frac{1}{6} \mathcal{M} v^{\prime} u+\frac{1}{6} \mathcal{M} w+\frac{1}{3} v \mathcal{J}+\frac{1}{6} v u \mathcal{M}^{\prime}, \\
\xi^{02}= & -\frac{1}{2} r v^{\prime \prime}+\frac{1}{6} v^{(4)} u+\frac{1}{6} w^{\prime \prime \prime}-\frac{7}{12} v^{\prime} u \mathcal{M}{ }^{\prime}-\frac{5}{12} \mathcal{M} v^{\prime \prime} u-\frac{1}{6} \mathcal{M}^{\prime} w-\frac{5}{12} \mathcal{M} w^{\prime}-\frac{5}{6} v^{\prime} \mathcal{J} \\
& -\frac{1}{3} v \mathcal{J}^{\prime}-\frac{1}{6} v u \mathcal{M}^{\prime \prime}+\frac{1}{2} \mathcal{M} r v, \\
\xi^{11}= & w+u v^{\prime}, \\
\xi^{12}= & r v-\frac{1}{2} v^{\prime \prime} u-\frac{1}{2} w^{\prime}, \\
\Lambda^{00}= & \frac{1}{6} v^{(4)}-\frac{1}{6} \mathcal{M}{ }^{\prime \prime} v-\frac{7}{12} \mathcal{M}^{\prime} v^{\prime}-\frac{2}{3} \mathcal{M} v^{\prime \prime}+\frac{1}{4} \mathcal{M}^{2} v+\mathcal{W} y, \\
\Lambda^{01}= & -\frac{1}{6} v^{\prime \prime}+\frac{1}{6} \mathcal{M} v, \\
\Lambda^{02}= & \frac{1}{6} v^{\prime \prime \prime}-\frac{1}{6} \mathcal{M}^{\prime} v-\frac{5}{12} \mathcal{M} v^{\prime}, \\
\Lambda^{12}= & -\frac{1}{2} v^{\prime} . \\
& \\
&
\end{aligned}
$$

\section{Appendix C: Global charges and its vanishing cosmological constant limit}

In the case of the asymptotically $\mathrm{AdS}_{3}$ conditions proposed in [14, 15], the expression for the global charges is given by

$$
Q=\frac{k l}{2 \pi} \int d \phi\left[\varepsilon^{+} \Xi_{+}+\varepsilon^{-} \Xi_{-}+4\left(\chi^{+} W_{+}+\chi^{-} W_{-}\right)\right],
$$

which, as expected, does not change for the gauge choice described in section IV. Here, the chiral functions $\varepsilon^{ \pm}\left(x^{ \pm}\right)$and $\chi^{ \pm}\left(x^{ \pm}\right)$correspond to the asymptotic symmetries spanned by the components of the Liealgebra-valued parameter along the generators $L_{ \pm 1}^{ \pm}$and $W_{ \pm 2}^{ \pm}$, respectively. In order to take the limit $l \rightarrow \infty$, it is convenient to express the charges according to the definitions in eqs. (34), (35), i.e.,

$$
Q=\frac{k}{4 \pi} \int d \phi[f \mathcal{M}+2 y \mathcal{N}+4(h \mathcal{W}+2 v \mathcal{Q})]
$$


so that the functions that parametrize the asymptotic symmetries become naturally redefined as

$$
f=\frac{l}{2}\left(\varepsilon^{+}+\varepsilon^{-}\right), \quad y=\frac{1}{2}\left(\varepsilon^{+}-\varepsilon^{-}\right), \quad h=\frac{l}{2}\left(\chi^{+}+\chi^{-}\right), \quad v=\frac{1}{2}\left(\chi^{+}-\chi^{-}\right),
$$

and hence, the chirality conditions now read

$$
\partial_{u} f=\partial_{\phi} y, \quad \partial_{u} y=\frac{1}{l^{2}} \partial_{\phi} f, \quad \partial_{u} h=\partial_{\phi} v, \quad \partial_{u} v=\frac{1}{l^{2}} \partial_{\phi} h
$$

Note that for $l \rightarrow \infty$, the latter conditions imply that

$$
y=y(\phi), \quad f=\epsilon(\phi)+u y^{\prime}, \quad v=v(\phi), \quad h=w(\phi)+u v^{\prime},
$$

and therefore, by virtue of the corresponding relationship of the functions $\mathcal{M}, \mathcal{N}, \mathcal{W}$ and $\mathcal{Q}$, in eq. (38), the global charges (C1) reduce to ours in eq. (14) in the vanishing cosmological constant limit.

[1] E. S. Fradkin and M. A. Vasiliev, "On the Gravitational Interaction of Massless Higher Spin Fields," Phys. Lett. B 189, 89 (1987).

[2] M. A. Vasiliev, "Consistent equation for interacting gauge fields of all spins in (3+1)-dimensions," Phys. Lett. B 243, 378 (1990).

[3] M. A. Vasiliev, "Nonlinear equations for symmetric massless higher spin fields in (A)dS(d)," Phys. Lett. B 567, 139 (2003) [hep-th/0304049].

[4] I. R. Klebanov and A. M. Polyakov, "AdS dual of the critical O(N) vector model," Phys. Lett. B 550 (2002) 213 [hep-th/0210114].

[5] M. R. Gaberdiel and R. Gopakumar, "An $A d S_{3}$ Dual for Minimal Model CFTs," Phys. Rev. D 83, 066007 (2011) [arXiv:1011.2986 [hep-th]].

[6] M. A. Vasiliev, "Higher spin gauge theories in four-dimensions, three-dimensions, and two-dimensions," Int. J. Mod. Phys. D 5, 763 (1996) [hep-th/9611024].

[7] X. Bekaert, S. Cnockaert, C. Iazeolla and M. A. Vasiliev, "Nonlinear higher spin theories in various dimensions," hep-th/0503128.

[8] X. Bekaert, N. Boulanger and P. Sundell, "How higher-spin gravity surpasses the spin two barrier: no-go theorems versus yes-go examples," Rev. Mod. Phys. 84, 987 (2012) [arXiv:1007.0435 [hep-th]].

[9] A. Sagnotti, "Notes on Strings and Higher Spins," arXiv:1112.4285 [hep-th].

[10] M. R. Gaberdiel and R. Gopakumar, "Minimal Model Holography," J. Phys. A 46, 214002 (2013) [arXiv:1207.6697 [hep-th]].

[11] M. Ammon, M. Gutperle, P. Kraus and E. Perlmutter, "Black holes in three dimensional higher spin gravity: A review," arXiv:1208.5182 [hep-th].

[12] M. P. Blencowe, "A Consistent Interacting Massless Higher Spin Field Theory In D = (2+1)," Class. Quant. Grav. 6, 443 (1989).

[13] E. Bergshoeff, M. P. Blencowe and K. S. Stelle, "Area Preserving Diffeomorphisms And Higher Spin Algebra," Commun. Math. Phys. 128, 213 (1990).

[14] M. Henneaux and S. -J. Rey, "Nonlinear $W_{\text {infinity }}$ as Asymptotic Symmetry of Three-Dimensional Higher Spin Anti-de Sitter Gravity," JHEP 1012, 007 (2010) [arXiv:1008.4579 [hep-th]].

[15] A. Campoleoni, S. Fredenhagen, S. Pfenninger and S. Theisen, "Asymptotic symmetries of three-dimensional gravity coupled to higher-spin fields," JHEP 1011, 007 (2010) [arXiv:1008.4744 [hep-th]].

[16] H. Afshar, A. Bagchi, R. Fareghbal, D. Grumiller and J. Rosseel, "Higher spin theory in 3-dimensional flat space," arXiv:1307.4768 [hep-th].

[17] J. D. Brown and M. Henneaux, "Central Charges in the Canonical Realization of Asymptotic Symmetries: An Example from Three-Dimensional Gravity," Commun. Math. Phys. 104, 207 (1986).

[18] A. Ashtekar, J. Bicak and B. G. Schmidt, "Asymptotic structure of symmetry reduced general relativity," Phys. Rev. D 55, 669 (1997) [gr-qc/9608042].

[19] G. Barnich and G. Compere, "Classical central extension for asymptotic symmetries at null infinity in three spacetime dimensions," Class. Quant. Grav. 24, F15 (2007) [gr-qc/0610130].

[20] G. Barnich and C. Troessaert, "Aspects of the BMS/CFT correspondence," JHEP 1005, 062 (2010) [arXiv:1001.1541 [hep-th]]. 
[21] G. Barnich, A. Gomberoff and H. A. Gonzalez, "The Flat limit of three dimensional asymptotically anti-de Sitter spacetimes," Phys. Rev. D 86, 024020 (2012) [arXiv:1204.3288 [gr-qc]].

[22] A. Bagchi, "Correspondence between Asymptotically Flat Spacetimes and Nonrelativistic Conformal Field Theories," Phys. Rev. Lett. 105, 171601 (2010).

[23] A. Campoleoni, S. Fredenhagen and S. Pfenninger, "Asymptotic W-symmetries in three-dimensional higherspin gauge theories," JHEP 1109, 113 (2011) [arXiv:1107.0290 [hep-th]].

[24] M. R. Gaberdiel and T. Hartman, "Symmetries of Holographic Minimal Models," JHEP 1105, 031 (2011) [arXiv:1101.2910 [hep-th]].

[25] T. Regge and C. Teitelboim, "Role of Surface Integrals in the Hamiltonian Formulation of General Relativity," Annals Phys. 88, 286 (1974).

[26] J. D. Brown and M. Henneaux, "On The Poisson Brackets Of Differentiable Generators In Classical Field Theory," J. Math. Phys. 27, 489 (1986).

[27] P. Kraus, "Lectures on black holes and the $\operatorname{AdS}(3)$ / CFT(2) correspondence," Lect. Notes Phys. 755, 193 (2008) [hep-th/0609074].

[28] E. Ayon-Beato, C. Martinez, R. Troncoso and J. Zanelli, "Gravitational Cheshire effect: Nonminimally coupled scalar fields may not curve spacetime," Phys. Rev. D 71, 104037 (2005) [hep-th/0505086].

[29] A. Castro, R. Gopakumar, M. Gutperle and J. Raeymaekers, "Conical Defects in Higher Spin Theories," JHEP 1202, 096 (2012) [arXiv:1111.3381 [hep-th]].

[30] A. Campoleoni, T. Prochazka and J. Raeymaekers, "A note on conical solutions in 3D Vasiliev theory," JHEP 1305, 052 (2013) [arXiv:1303.0880 [hep-th]].

[31] A. Campoleoni and S. Fredenhagen, "On the higher-spin charges of conical defects," arXiv:1307.3745 [hep-th].

[32] G. Barnich and H. A. Gonzalez, "Dual dynamics of three dimensional asymptotically flat Einstein gravity at null infinity," JHEP 1305, 016 (2013) [arXiv:1303.1075 [hep-th]].

[33] G. Barnich, A. Gomberoff and H. A. Gonzalez, "BMS3 invariant two dimensional field theories as flat limit of Liouville," Phys. Rev. D 87:124032, (2013) [arXiv:1210.0731 [hep-th]].

[34] O. Coussaert, M. Henneaux and P. van Driel, "The Asymptotic dynamics of three-dimensional Einstein gravity with a negative cosmological constant," Class. Quant. Grav. 12, 2961 (1995) [gr-qc/9506019]. 